\title{
PREVISIONE DELLA PROPAGAZIONE DI SARS-COV-2 NELLO STATO DI AMAPÁ, AMAZZONIA, BRASILE, MEDIANTE MODELLAZIONE MATEMATICA
}

\section{ARTICOLO ORIGINALE}

DIAS, Neylan Leal', SILVA, Edcarlos Vasconcelos $\mathrm{da}^{2}$, PIRES, Marcelo Amanajas ${ }^{3}$, CHAVES, Daniel ${ }^{4}$, SANADA, Katsumi Letra ${ }^{5}$, FECURY, Amanda Alves ${ }^{6}$, DIAS, Cláudio Alberto Gellis de Mattos $^{7}$, OLIVEIRA, Euzébio de ${ }^{8}$, DENDASCK, Carla Viana $^{9}$, LEAL, Simone Delphim ${ }^{10}$

DIAS, Neylan Leal. Et al. Previsione della propagazione di SARS-CoV-2 nello Stato di Amapá, Amazzonia, Brasile, mediante modellazione matematica. Revista Científica Multidisciplinar Núcleo do Conhecimento. Anno 05, Ed. 05, Vol.06, pagg. 73-95. Maggio 2020. ISSN: 2448-0959, Link di accesso: https://www.nucleodoconhecimento.com.br/salute/previsione-della-propagazione, DOI: 10.32749/nucleodoconhecimento.com.br/salute/previsione-della-propagazione

\footnotetext{
${ }^{1}$ Matematico, dottorando in Ingegneria Meccanica - FEB / UNESP-SP. Professore e ricercatore presso il Corso di Matematica presso I'Università Federale di Amapá (UNIFAP).

2 Matematico, studente di dottorato in sanità pubblica - FIOCRUZ / RJ. Professore del Corso di Matematica presso l'Università Federale di Amapá (UNIFAP).

${ }^{3}$ Fisico, Master in Fisica (UFF), studente di dottorato presso il Centro brasiliano per la ricerca fisica (CBPF).

${ }^{4}$ Storico, dottore in storia comparata, professore e ricercatore del corso di storia, dei corsi di laurea in sviluppo regionale e in proprietà intellettuale e trasferimento tecnologico per l'innovazione, Università federale di Amapá (UNIFAP).

${ }^{5}$ Letteratura, Laurea Magistrale in Scienze dell'Educazione (UNIFAL) presso l'Istituto Federale di Amapá (IFAP).

${ }^{6}$ Biomedico, PhD in Malattie Tropicali, Professore e ricercatore del Corso di Medicina presso I'Università Federale di Amapá (UNIFAP).

${ }^{7}$ Biologo, dottore in teoria e ricerca del comportamento, professore e ricercatore presso il Facoltà di Chimica, Campus Macapá, Istituto Federale di Amapá (IFAP).

${ }^{8}$ Biologo, PhD in Malattie topiche, Professore e ricercatore del Corso di Educazione Fisica presso I'Università Federale del Pará (UFPA).

${ }^{9}$ Teologo, PhD in Psicoanalisi, ricercatore presso il Centro Ricerche e Studi Avanzati - CEPA.

${ }^{10}$ Matematica, PhD in Modellazione Computazionale, Professore e ricercatore del Corso di Matematica presso I'Università Federale di Amapá (UNIFAP).
}

RC: 80639

Disponibile in: https://www.nucleodoconhecimento.com.br/salute/previsione-dellapropagazione 


\section{ASTRATTO}

Questo articolo presenta un'analisi della diffusione di SARS-CoV-2 in Amapá utilizzando tre approcci. Nella prima, il modello ICL per la pandemia applicata al Brasile è stato utilizzato per implementare una proiezione lineare comparativa per la popolazione di Amapá. II secondo approccio è stato sviluppato con la soluzione a breve termine del modello SIR standard, dove è stato dimostrato che il comportamento esponenziale tipico descrive in modo soddisfacente i dati per le prime settimane dell'epidemia, ma subito dopo, ci sono prime discrepanze dovute a un improvviso rallentamento dell'evoluzione temporale numero di casi dovuti a misure di isolamento. Questo nuovo regime è opportunamente descritto con il terzo approccio che si basa sul modello vSIR che è una variante del modello SIR. I risultati presentati consentono, da un lato, una migliore comprensione degli scenari già affrontati dalla popolazione e, dall'altro, forniscono proiezioni di breve periodo che saranno costantemente aggiornate sul collegamento [11].

Parole chiave: COVID-19, pandemia, modello matematico, vSIR, SARS-CoV-2.

\section{INTRODUZIONE}

II Coronavirus è una famiglia di virus che provocano infezioni respiratorie che, sebbene registrati dal 1937, solo il 31 dicembre 2019, dopo casi registrati in Cina, è stato scoperto e identificato il nuovo coronavirus come causa della malattia chiamata COVID-19 che è provocata. dal coronavirus SARS-CoV-2 (MINISTÉRIO DA SAÚDE, 2020b).

Secondo l'Organizzazione Mondiale della Sanità (OMS), circa l'80\% dei casi può essere asintomatico e il restante $20 \%$ richiede spesso cure ospedaliere e una piccola parte - circa il 5\% - di questi va alle unità di terapia intensiva a causa di una grave insufficienza respiratoria. (OMS, 2020).

RC: 80639

Disponibile in: https://www.nucleodoconhecimento.com.br/salute/previsione-dellapropagazione 
Questo fatto si riflette nella condizione di trasmissione comunitaria installata a livello nazionale in Brasile, dove anche considerando i bassi test del Paese e la costante revisione dei decessi da parte del COVID-19, il tasso medio di mortalità si aggira intorno al $6 \%$ e arriva la proiezione dei casi. il mese di maggio, probabilmente, a sei cifre, si è diffuso principalmente nella regione sud-orientale. In questo senso, la situazione nella regione del Nord è di notevole impatto; a fine aprile si registrava circa il $12 \%$ dei casi nazionali in numero assoluto, il che diventa ancora più drammatico considerando la proporzionalità demografica tra le cinque macroregioni nazionali (BATISTA et al, 2020b).

A metà maggio, sia l'incidenza che la mortalità per 100mila abitanti al Nord $(245,7)$ erano il doppio dei tassi delle regioni più avanzate in questo stesso quadro, come mostrato nella Tabella 1 (OMS, 2020a).

Tabella 1. Riepilogo di casi, decessi, incidenza e mortalità di covid-19 Brasile e regioni.

\begin{tabular}{ccccc}
\hline & Casos & Óbitos & $\begin{array}{c}\text { Incidência } \\
(\mathbf{1 0 0} \mathrm{mil} \\
\text { hab })\end{array}$ & $\begin{array}{c}\text { Mortalidade } \\
(\mathbf{1 0 0} \mathrm{mil} \\
\text { hab })\end{array}$ \\
\hline Região Sudeste & 10.797 & 349 & 36 & 1,2 \\
Região Sudeste & 96.938 & 7938 & 108,6 & 9 \\
Região Centro-Oeste & 7.531 & 171 & 4602 & 1 \\
Região Nordeste & 81.537 & 4.673 & 142,9 & 8,2 \\
Região Norte & 45.277 & 2.987 & 245,7 & 16,2 \\
\hline Brasil & 242.080 & 16.118 & 114,7 & 7,7 \\
\hline
\end{tabular}

Fonte: Ministero della Salute (2020).

RC: 80639

Disponibile in: https://www.nucleodoconhecimento.com.br/salute/previsione-dellapropagazione 
Nel caso di Amapá, come si può vedere in Tabella 2, nello stesso periodo i dati presentati hanno seguito la tendenza al rialzo, con una media simile a quella regionale sulla mortalità (14,1 dell'AP x 16,2 al Nord), ma con allarmanti numeri per quanto riguarda l'incidenza dei casi (467,3 dell'AP x 245,7 al Nord). Pertanto, c'è urgente bisogno di un'attenzione specifica alla contraddizione visibile tra i modelli di incidenza e mortalità nella regione alla luce della realtà nazionale, dato che, considerando la densità demografica della regione del Nord e il numero di casi pro capite , 5 città del nord erano tra le 20 città con il più alto tasso di mortalità alla fine di aprile (BATISTA et al, 2020b).

Tabella 2. Riepilogo dei casi, decessi, incidenza e mortalità del covid-19 Nord Region e stati

\begin{tabular}{ccccc}
\hline Estados & Casos & Óbitos & $\begin{array}{c}\text { Incidência } \\
(\mathbf{1 0 0} \mathrm{mil} \\
\text { hab })\end{array}$ & $\begin{array}{c}\text { Mortalidade } \\
(100 \mathrm{mil} \\
\text { hab })\end{array}$ \\
\hline AP & 3.952 & 119 & 467,3 & 14,1 \\
RO & 1.963 & 74 & 110,5 & 4,2 \\
AC & 1.997 & 60 & 226,4 & 6,8 \\
PA & 13.864 & 1.239 & 161,2 & 14,4 \\
RR & 1.791 & 51 & 295,7 & 8,4 \\
TO & 1.382 & 31 & 87,9 & 2 \\
AM & 20.328 & 1.413 & 490,5 & 34,1 \\
\hline Norte & 45.277 & 2.987 & 245,7 & 16,2 \\
\hline
\end{tabular}

Fonte: Ministero della Salute (2020).

RC: 80639

Disponibile in: https://www.nucleodoconhecimento.com.br/salute/previsione-dellapropagazione 
Data la configurazione dei sistemi sanitari pubblici nazionali e regionali, ci sono diversi fattori che, a loro volta, sono tipicamente legati ai paesi in via di sviluppo come il Brasile - come il basso numero di letti con respiratori o capacità di trattamento intensivo, il ritardo tecnologico che influenza la qualità dei test, assenza di dispositivi di protezione individuale (EPI), carenze nei sistemi sanitari delle città, saturazione del numero di persone per dormitorio in abitazioni in regioni ad altissima densità demografica e l'inesperienza brasiliana con situazioni di reclusione e / o limitazione del traffico - rendono le proiezioni epidemiologiche e infettive su COVID19 ancora più incerte. Tali incertezze devono osservare, anche se con la dovuta attenzione, la modellazione macroscopica che mira alla proiezione più vicina possibile 01 (PIRES; CARVALHO; XAVIER, 2020).

\section{COVID-19 IN AMAPÁ E NELLA REGIONE METROPOLITANA DELLO STATO}

Con l'arrivo del Coronavirus ad Amapá il 25 marzo 2020, la cui regione metropolitana comprende una popolazione secondo I'IBGE di circa 845.000 abitanti, si sviluppa su 16 comuni con un tasso di crescita demografica del 2,02\% (IBGE, 2020).

È importante avere un quadro il più accurato possibile, in questo senso osservare i modelli comportamentali di circolazione delle persone, l'igiene e la cura con la salute ospedaliera possono influenzare la diffusione del COVID-19 ad Amapá, considerando anche i modelli comportamentali dei valori assoluti Di casi notificati (SVS, 2020).

Tangenti da una diffusione sfrenata del nuovo coronavirus ad Amapá, pensiamo alle famiglie alienate dalla forza lavoro e in isolamento sociale, a cui è stato impedito di svolgere la loro professione. In queste condizioni, l'unica soluzione è aspettare che la malattia passi, provocando altri problemi come ansia, depressione, stress posttraumatico, irritabilità, tra gli altri impatti psicologici della quarantena. Per la loro risposta agli impatti negativi, le persone soffrivano anche di fatti predittivi come:

RC: 80639

Disponibile in: https://www.nucleodoconhecimento.com.br/salute/previsione-dellapropagazione 
paura, tristezza, senso di colpa, confusione, per non essere in grado di trovare da sole una soluzione lavorativa o per hobby. Parte di questi contesti psicologici citati è percettibile in tutte le classi e livelli sociali dello Stato di Amapá, a cominciare principalmente dagli operatori sanitari che lavorano e sono sempre in prima linea in centri di cura inadeguati e insufficienti per anni vissuti dietro un sistema politico allarmante della disuguaglianza democratica (BROOKS, 2020).

Nella catalogazione che esiste ai vari livelli dei gruppi sociali urbani e periferici nello stato di Amapá, una città come Macapá e Santana sono i due comuni in cui la malattia è più concentrata. Secondo la Soprintendenza per la sorveglianza sanitaria SVS, questa dichiarazione ricercata si è basata sul periodo dal 14 al 27 aprile, mostrando sempre le caratteristiche di concentrazione del COVID-19 nel centro della città di Macapá, dove tutto è iniziato, questo è dovuto a persone che viaggiato all'estero dello stato e tornato. Si conclude che quindi l'effetto dell'espansione della contaminazione è dato dalle persone importate che chiameremo Class A e B (Socioeconomic Classification Criterion). In parte contraria alle conferme dell'epicentro sotto pressione del COVID-19 nel centro di Macapá, c'è Santana che ratifica le sue statistiche di concentrazione in tutte le possibili aree urbane e periferiche di sfollamento intercity necessario per motivi familiari, residenziali o meno (contatto diretto), anche con le misure di contenimento applicate dalle barriere sanitarie. Questo panorama sarà presentato dal gruppo di persone di Classe C, D, E, inquadrate nella dipendenza dall'uso del trasporto collettivo e privato (SVS, 2020).

In questa idea commentata sopra, abbiamo integrato un modello quadro per le relazioni di classi o gruppi sociali di isolamento, misurato dal reddito e dal benessere tra le persone Amapá (IN LOCO, 2020).

RC: 80639

Disponibile in: https://www.nucleodoconhecimento.com.br/salute/previsione-dellapropagazione 
Tabella 3. Relazione comparata di isolamento sociale per gruppo socioeconomico.

\begin{tabular}{|c|c|c|c|}
\hline $\begin{array}{c}\text { Grupos Sociais por } \\
\text { Classe }\end{array}$ & Isolamento Social & Bem Estar Social & Cidade \\
\hline$A \in B$ & $\begin{array}{l}40 \% \text { em casa } \\
\text { respeitando o } \\
\text { decreto }\end{array}$ & $\begin{array}{l}\text { Variante conforme o } \\
\text { comportamento da } \\
\text { equidade do início da } \\
\text { pandemia }\end{array}$ & Macapá \\
\hline C, DeE & $\begin{array}{c}60 \% \text { não respeitando } \\
\text { o decreto de } \\
\text { isolamento social }\end{array}$ & $\begin{array}{l}\text { Invariante conforme } \\
\text { até um ponto } \\
\text { convergente das } \\
\text { informações de } \\
\text { prevenção dada do } \\
\text { início da pandemia }\end{array}$ & $\begin{array}{c}\text { Macapá, Santana e } \\
\text { demais Municípios } \\
\text { do Estado }\end{array}$ \\
\hline
\end{tabular}

Lo scenario del tavolo è solo una piccola dimostrazione della realtà comportamentale sociale della popolazione Amapá, per quanto riguarda il valore della loro responsabilità di mantenere il proprio benessere dalla durata del decreto stabilito dalle agenzie del governo statale. Amata in casa dalle notizie serializzate dalla pandemia, la foto mostra il $40 \%$ delle classi A e B disposte a soddisfare l'isolamento, parte di questo adempimento è dovuto a queste persone che hanno aziende, posti di lavoro e stipendi fissi di dipendenti pubblici in carriera. Contrariamente alle altre classi sociali rimanenti $C, D$ ed $E$, esiste un criterio dello standard salariale combinato tra parti contributive del reddito fisso familiare (dipendenza finanziaria) e lavori temporanei formali e informali per il benessere atteso, indipendentemente dal indipendentemente dal fatto che sia o meno un funzionario pubblico o di formazione accademica. Questo è il motivo della crescente violazione dell'isolamento sociale, che raggiunge il $60 \%$ con una tendenza a crescere ancora di più durante i mesi in corso (IBGE-AMAPÁ, 2020).

RC: 80639

Disponibile in: https://www.nucleodoconhecimento.com.br/salute/previsione-dellapropagazione 
A seguito della convalida dei fatti in questione da parte di ciascuna classe sociale intervenuta per ragioni pluraliste, la figura 1 è delineata in una dichiarazione generale che mostra l'indice di irresponsabilità della popolazione riguardo all'isolamento sociale nello Stato di Amapá città di Macapá Capitale e in altri Comuni nel periodo di gennaio Dal 30 al 14 maggio 2020 con tendenza a variare, dividere o regredire (IN LOCO, 2020).

Figura 1. Indice di isolamento sociale per Amapá dal 30 gennaio al 14 maggio.

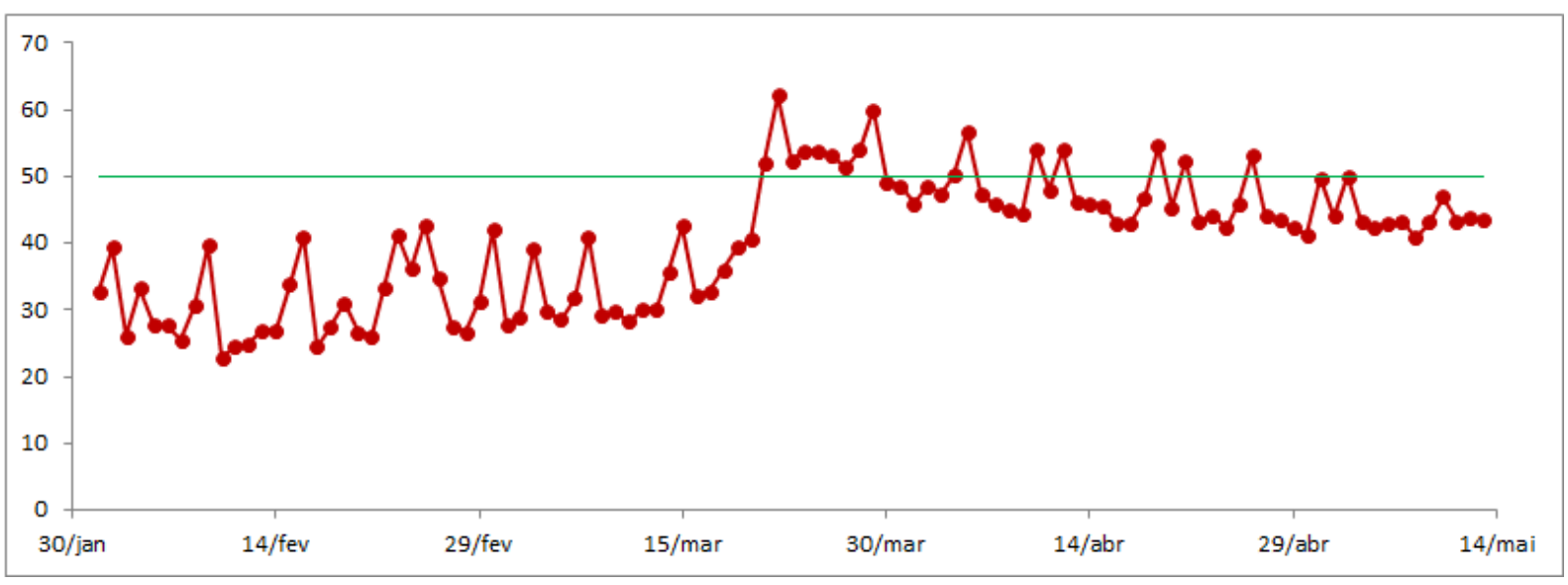

Fonte: In Loco (2020).

Analisi statistiche di isolamento sociale, sono ancora varianti incerte per quanto concordato in pratica dalla popolazione Amapá, la variazione è ancora attesa dal periodo grafico fino al 14 maggio 2020, considerato il compimento del rimanere a casa e partire solo se necessario o di emergenza. Le azioni delle barriere di ispezione sanitaria saranno la base per controllare l'intenso flusso di persone in giro per la città. $E$ anche se ci sono abbastanza casi sintomatici sospetti del virus così informato, il risultato incontrollabile atteso è ancora monitorato. (SVS, 2020)

\section{OBBIETTIVO}

Questa ricerca mira ad analizzare l'incidenza dei casi confermati di COVID-19 in Amapá / AP utilizzando metodi statistici e modelli matematici al fine di comprendere

RC: 80639

Disponibile in: https://www.nucleodoconhecimento.com.br/salute/previsione-dellapropagazione 
le dinamiche di contagio del virus, fornendo informazioni per promuovere una strategia di combattimento più efficace contro il covid-19 .

Tra le altre specifiche di guida, si intende verificare il comportamento forzato di restrizione sociale nell'ambiente pubblico e domestico, data come una delle condizioni assistenziali prioritarie per la proliferazione di riduzione del contagio da parte del Coronavirus.

\section{METODOLOGIA}

In generale, sono stati impiegati due approcci di modellazione per ottenere insights sull'evoluzione epidemica correlata a Sars-CoV-2. Da un lato, c'è uno sforzo per sviluppare modelli sofisticati che tengano conto di diverse variabili che possono descrivere le dinamiche epidemiche. Un tale approccio è stato utilizzato, ad esempio, in Zhang (2020) con un sistema di 15 equazioni differenziali. D'altra parte, è stata adottata anche una visione matematica incentrata sulle principali caratteristiche epidemiche del processo contagioso di successo per la curva epidemica del coronavirus attraverso versioni primarie o estese dei modelli SIR (TODA, 2020; KATUL, 2020; ZHONG, 2020).

II modello epidemiologico SIR proposto da Kermack e McKendrick nel 1927 (HETHCOTE, 2000) è uno dei modelli classici utilizzati per descrivere l'evoluzione del processo di contagio delle malattie infettive. Questo modello composto da equazioni differenziali impiega la strategia compartimentale e divide, in relazione alla malattia, la popolazione in individui che possono contrarre, individui che possono trasmettere e individui che sono guariti e non sono soggetti a nuova contaminazione chiamata sensibile $(S)$, infetta $(I)$ e recuperato $(R)$ rispettivamente.

RC: 80639

Disponibile in: https://www.nucleodoconhecimento.com.br/salute/previsione-dellapropagazione 
Matematicamente, un modello SIR può essere rappresentato utilizzando le seguenti equazioni differenziali:

$$
\begin{aligned}
& \frac{d S}{d t}=-\frac{\beta I S}{N}, \\
& \frac{d I}{d t}=\frac{\beta I S}{N}-\gamma I, \\
& \frac{d R}{d t}=\gamma I,
\end{aligned}
$$

Dove $\beta$ è il tasso di trasmissione della malattia, $\gamma$ è il tasso di recupero degli individui, entrambi sono parametri positivi e caratteristici dei dati in analisi.

Al fine di ottimizzare e stimare questi parametri in modo che i casi riportati e simulati siano approssimativamente uguali, i valori iniziali vengono utilizzati per le variabili a tre stati $S(0), I(0)$ e $R(0)$.

II valore iniziale $S(0)$ è la comunità di popolazione colpita dall'epidemia, mentre $I(0)$ è il numero di casi confermati che possono avere qualsiasi valore, ma non zero. Possiamo impostare $R(0)$ a zero se lo spread e gli orari di inizio della simulazione sono gli stessi. La velocità di trasmissione si riduce monotonicamente nel tempo (HARKO, 2014).

Risolvendo il sistema (1) determiniamo la funzione di soluzione per la popolazione suscettibile e otteniamo (BATISTA, 2020).

$$
S=S_{0} \exp \left[-\frac{\beta}{N \gamma}\left(R-R_{0}\right)\right]
$$

RC: 80639

Disponibile in: https://www.nucleodoconhecimento.com.br/salute/previsione-dellapropagazione 
Tra i parametri rilevanti utilizzati nei modelli epidemiologici come il SIR c'è la riproducibilità di base fornita da $R_{0}$ (nota come velocità di riproduzione) definita da $R_{0}$ $=\beta / y$. II tasso $R_{0}$ è influenzato, tra diversi fattori, dal numero di persone suscettibili nella popolazione con cui i pazienti affetti sono in contatto e può essere descritto come il numero medio di persone infettate da un singolo individuo (DELAMATER et al, 2019 ).

Secondo Ghani e Fergunson (2020), il valore $R_{0}$ per COVID-19 è variato tra 1 e 7 (più precisamente da 1,1 a 6,95) durante lo sviluppo dell'epidemia. Come indicato da Delamater et al (2019), un $R_{0}<1$ mostra che il numero di individui infetti diminuisce con il tempo e l'epidemia tende a sradicare, per valori di $R_{0}>1$ si presume che l'epidemia debba persistere nella popolazione a lungo termine.

Quindi, prendendo il limite quando $t$ tende all'infinito, il numero di persone suscettibili ottenuto è:

$$
S_{\infty}=S_{0} \exp \left[-\frac{\beta}{N \gamma}\left(R_{\infty}-R_{0}\right)\right]
$$

Dov'è l'importo finale recuperato. Considerando che il numero finale di persone infette è zero, abbiamo:

$$
N=S_{\infty}+R_{\infty}
$$

(03) otteniamo

$$
R_{\infty}=N-S_{0} \exp \left[-\frac{\beta}{N \gamma}\left(R_{\infty}-R_{0}\right)\right]
$$

RC: 80639

Disponibile in: https://www.nucleodoconhecimento.com.br/salute/previsione-dellapropagazione 
Supponendo che i dati disponibili siano una serie temporale del numero totale di casi $C=R+I$ parametri ei valori iniziali sono definiti minimizzando la differenza tra $\mathrm{i}$ valori correnti ei numeri dei casi calcolati:

$$
\left\|C_{t}-\hat{C}_{t}\left(\beta, \gamma, S_{0}\right)\right\|^{2}=\min
$$

dove $C_{t}=\left(C_{1}, C_{2}, \ldots, C_{n}\right)$ è il numero di casi al tempo $t=1,2, \ldots, n$ e il vettore corrisponde ai valori stimati negli stessi momenti valori. La minimizzazione in questione è stata ottenuta dalla funzione fminsearch di MATLAB secondo Batista (2020).

Sottolineiamo che nel suddetto approccio ci sono tre parametri da stimare, vale a dire $\{\beta, \gamma, N\}$ dove $N$ è la dimensione effettiva della popolazione. Indichiamo questa variante con l'acronimo vSIR per differenziarla dal modello SIR standard dove $N$ è la dimensione totale della popolazione che è una quantità definita a priori. A livello computazionale, la soluzione numerica del sistema di equazioni vSIR è implementata con il software MATLAB utilizzando il metodo Runge-Kutta del quarto ordine esplicito (BATISTA, 2020).

Tutte le analisi con il modello matematico di questo studio sono state condotte con i dati dei casi confermati di covid-19 per lo Stato di Amapá, messi a disposizione dal Dipartimento della Salute dello Stato e dal Ministero della Salute del Brasile, entrambi online, nel caso di dati secondari, per questo motivo, non è stato necessario registrare la ricerca in un comitato etico.

RC: 80639

Disponibile in: https://www.nucleodoconhecimento.com.br/salute/previsione-dellapropagazione 


\section{RISULTATI E DISCUSSIONI}

\section{ANALISI DEL PROGETTO DEL MODELLO IMPERIALE DEL COLLEGE LONDON}

Presentiamo in questo argomento alcune proiezioni locali realizzate con il modello Imperial College of London (ICL), che hanno cercato di guidare le politiche pubbliche di emergenza nel controllo epidemiologico-infettivo del COVID-19 per i paesi colpiti, ovviamente tutti i modelli devono essere attenti in un momento immediato all'espansione transoceanica della pandemia, considerando i fattori aggravanti che si verificano in ciascuna regione.

Pertanto, il "Modello ICL" descrive i cinque scenari come segue:

A) Un'epidemia assoluta - uno scenario in cui non viene intrapresa alcuna azione.

B) Mitigazione, inclusa la distanza sociale a livello di popolazione - valutiamo la massima riduzione nella scala finale dell'epidemia che può essere ottenuta attraverso una riduzione uniforme della velocità con cui gli individui comunicano, ad eccezione della completa soppressione.

C) Mitigazione, inclusa una maggiore distanza sociale dagli anziani - come (B), ma con individui di 70 anni o più, riducendo i loro tassi di contatto sociale del $60 \%$.

D ed E) Soppressione: esploriamo diversi trigger epidemiologici (morti per 100.000 abitanti) per l'attuazione di un distacco sociale intensivo su larga scala (modellato come una riduzione del $75 \%$ dei tassi di contatto interpersonale) con l'obiettivo di sopprimere rapidamente la trasmissione e ridurre al minimo casi a termine e decessi.

Per questi scenari, non produciamo stime finali delle dimensioni, ma illustriamo il loro impatto su configurazioni rappresentative. Per Tardia si considera condurre test di massa, isolare casi positivi e monitorare persone vicine a quelle diagnosticate da 1,6

RC: 80639

Disponibile in: https://www.nucleodoconhecimento.com.br/salute/previsione-dellapropagazione 
morti ogni 100mila abitanti a settimana. Per Precoce, le stesse misure di prima, ma adottate da 0,2 morti ogni 100mila abitanti a settimana (GHANI; FERGUSON, 2020).

Gli scenari esposti da ICL per una popolazione in Brasile di oltre 200 milioni di persone attualmente, sono estrapolati (per la premessa fondamentale di minimizzare gli impatti per la popolazione) sono i seguenti: Scenario A - Nessuna misura di mitigazione; Scenario B - Con distanza sociale dallintera popolazione. Nello Scenario $\mathrm{A}$, la situazione brasiliana è spericolata e, a seconda dello scenario, con percentuali di persone infette che vanno dall' $88 \%$ e scendono fino al $5 \%$ della popolazione, fino a un numero di morti che va dall' $1 \%$ allo $0,02 \%$. C'è anche un terzo scenario - C - che lavora con la distanza sociale e rafforza la distanza tra gli anziani. Infine, vengono considerati gli scenari $\mathrm{D}$ ed $\mathrm{E}$, corrispondenti rispettivamente alla soppressione tardiva e precoce, essendo tra gli scenari catastrofici, quelli di minore distruzione quantitativa, come si può vedere di seguito.

Abbiamo costruito, per amor di apprezzamento, la seguente proiezione nazionale, secondo il Modello ICL.

RC: 80639

Disponibile in: https://www.nucleodoconhecimento.com.br/salute/previsione-dellapropagazione 
Tabella 4. Proiezioni per il Brasile secondo il modello ICL.

\begin{tabular}{cccccc} 
& A & B & $\begin{array}{c}\text { C } \\
\text { Mitigação com } \\
\text { ênfase em } \\
\text { idosos }\end{array}$ & Supressão tardia & $\begin{array}{c}\text { Supressão } \\
\text { precoce }\end{array}$ \\
\hline $\begin{array}{c}\text { População } \\
\text { Infectada }\end{array}$ & $188 \mathrm{Mi}$ & $122 \mathrm{Mi}$ & $120 \mathrm{Mi}$ & $50 \mathrm{Mi}$ & $11 \mathrm{Mi}$ \\
$\begin{array}{c}\text { \% infectados } \\
\text { Mortes }\end{array}$ & 88 & 57 & 57 & 23 & 5 \\
$\begin{array}{c}\text { \% mortes } \\
\text { Necessitando } \\
\text { internação }\end{array}$ & $1.15 \mathrm{Mi}$ & 620.000 aprox & 530.000 aprox & 206.000 aprox & 44.000 aprox \\
$\begin{array}{c}\text { Necessitando } \\
\text { UTI }\end{array}$ & $1.5 \mathrm{Mi}$ & 830.000 aprox & 700.000 aprox & 97.000 aprox & 57.000 aprox \\
\hline
\end{tabular}

Fonte: prodotto dagli autori con dati di ricerca.

Alla fine di marzo, lo scenario attuale indicava un'accelerazione del numero di casi rilevati, circa una settimana dopo le tensioni intorno al confino, come si può vedere nella proiezione seguente. I dati del Ministero della Salute, consolidando le Segreterie Sanitarie di Stato, indicano 5.812 contagiati e 202 decessi nel $36^{\circ}$ giorno dopo il Primo Caso Confermato. Ci sono voluti 22 giorni fino al $500^{\circ}$ caso, e da quel momento in poi al $1000^{\circ}$ ci sono voluti solo 2 giorni, raddoppiando in tre giorni (2000) e raggiungendo il doppio del 2000 in 5 giorni. Negli ultimi 15 giorni di marzo, quindi, c'è stata una forte accelerazione (PRADO, M. et al., 2020).

È già noto che il Brasile sta vivendo uno scenario di sottostima dall'inizio della pandemia, con un tasso di notifica stimato in Amapá di circa l'11,2\%, come sottolineato in uno studio nella prima metà di aprile (PRADO et al., 2020). Questo fattore può essere osservato a causa dei problemi di pianificazione per lo 'scoppio' della crisi - nonostante sia stato previsto dall'ultima settimana di gennaio, quando la proliferazione ha iniziato a superare il continente asiatico - e del basso numero di test

RC: 80639

Disponibile in: https://www.nucleodoconhecimento.com.br/salute/previsione-dellapropagazione 
, a loro volta, sono per lo più applicati a individui sintomatici, trascurando l'enorme potenziale dei contaminanti asintomatici; cioè che sono già stati infettati dal virus, ma non avendo sintomi, si sentono a proprio agio per il traffico gratuito e migliorano la trasmissione. Andrebbero soppesati anche fattori come le tensioni dalla fine di marzo intorno a una parte significativa della popolazione che contesta il confinamento o minimizzarne gli effetti, rendendo un peso il carattere elastico e informale delle promozioni di libera circolazione. Precedente (PRADO et al., 2020).

Nel caso di Amapá, a marzo è stata osservata la possibile escalation, sebbene mantenuta in quella progressione in corso, per i casi autoctoni - già in trasmissione comunitaria (AMAPÁ, 2020a) - a causa delle battute d'arresto con l'autorizzazione ai test locali (autorizzati solo in 27 marzo - e con la riduzione del tempo di progressione dal 26 marzo. Per quanto riguarda il mese di marzo, tra il 19 marzo e il 24 marzo, abbiamo registrato 1 caso per 5 giorni; tra il 25 e il 26 marzo siamo passati al $2^{\circ}$ caso; tra 27 e 28 marzo, siamo passati al $3^{\circ}$; il 29 marzo, 6 casi; 30 marzo , 8 casi e 31 marzo, 10 casi. Ad aprile, il tasso osservato di casi è aumentato a rischio di mancanza di controllo ambientale (AMAPÁ, 2020a)

Considerando anche che le ultime proiezioni IBGE riguardano una popolazione di 845.000 persone stimate per Amapá (IBGE, 2020), se la modellizzazione ICL per lo stato è proiettata, in modo lineare rispetto allo scenario nazionale - che di per sé ne rappresenta già uno in più fattore di distorsione - avremmo il seguente scenario di previsione, severamente estrapolato per essere accumulato sull'intero primo ciclo epidemiologico di propagazione del COVID-19 di seguito:

RC: 80639

Disponibile in: https://www.nucleodoconhecimento.com.br/salute/previsione-dellapropagazione 
Tabella 5. Proiezione lineare comparativa del modello ICL p. COVID-19 ha presentato domanda in Brasile per Amapá.

\begin{tabular}{|c|c|c|c|c|c|}
\hline & & & & & \\
\hline & A & B & C & D & $\mathbf{E}$ \\
\hline & Năo mitigada & Mitigaçăo & $\begin{array}{c}\text { Mitigaçăo com } \\
\text { ênfase em } \\
\text { idosos }\end{array}$ & Supressăo tardia & $\begin{array}{c}\text { Supressăo } \\
\text { precoce }\end{array}$ \\
\hline $\begin{array}{l}\text { Populaçăo } \\
\text { Infectada }\end{array}$ & 743.000 aprox & 480.000 aprox & 480.000 aprox & 194.000 aprox & 42.000 aprox \\
\hline$\%$ infectados & 88 & 57 & 57 & 23 & 5 \\
\hline Mortes & 8.400 aprox & 2.500 aprox & 2.100 aprox & 845 & 160 \\
\hline$\%$ mortes & 1 & 0,3 & 0,25 & 0,1 & 0,02 \\
\hline $\begin{array}{l}\text { Necessitando } \\
\text { internaçăo }\end{array}$ & 22.000 aprox & 12.000 aprox & 11.000 aprox & 3.600 aprox & 1500 aprox \\
\hline
\end{tabular}

Fonte: prodotto dagli autori con dati di ricerca.

A causa del Decreto del Governo dello Stato di Amapá (GEA) n. 1414/2020, che ha promosso un'ampia riduzione delle attività economiche, sociali e culturali, e l'ampia adozione di azioni a favore del decentramento delle persone, scenario A è imminentemente scartato nell'estrapolato fintanto che l'isolamento è mantenuto. La preoccupazione fondamentale diventa, in modo cogente, il mantenimento dellisolamento con misure di contenimento intelligente dei flussi per evitare l'indisciplina sociale in relazione a questo, mitigare il più possibile gli schemi dello scenario $B$ e $C$ per avvicinarsi allo scenario realistico $D$, di soppressione tardiva - che renderebbe, in numero assoluto, la crisi navigabile ad Amapá, data la sua densità demografica e il suo contingente di popolazione. II motivo dell'ottimismo è a favore delle azioni rapide dei municipi metropolitani e principalmente del governo dello stato; tuttavia, le condizioni tecniche di notifica, nonché il livello quantitativo e

RC: 80639

Disponibile in: https://www.nucleodoconhecimento.com.br/salute/previsione-dellapropagazione 
qualitativo delle strutture e dei posti letto per il ricovero o la terapia intensiva, suggeriscono un'emergenza quando si conferma un proliferare di casi. Per questo motivo, il contenimento della trasmissione della comunità è risultato essere l'opzione migliore. Le previsioni presentate nella proiezione di un team congiunto della Fondazione Getúlio Vargas e della Fondazione Oswaldo Cruz (FGV/Fiocruz) puntano in modo convergente verso gli effetti positivi delle restrizioni sociali e di trasferimento combinate rispetto a una prossima ondata nazionale accumulata sull'intero primo periodo ciclo epidemiologico di propagazione del COVID-19 (ZORZETTO, 2020).

È importante ribadire la preoccupazione per gli ovvi adattamenti della modellistica ICL in relazione alle caratteristiche socio-culturali e socio-economiche del nostro Paese. Allo stesso tempo, i dati degli scenari di modellazione ICL sono numeri estrapolati e possono, in questo senso, costituire un'esagerazione. In tal senso, in modo preventivo, verificando la propensione per i prossimi giorni di moltiplicazione dei casi nazionali e l'impianto ancora attuale del sistema di sperimentazione locale e l'arrivo imminente di esami rapidi di prova - che rischiano di essere insufficienti e concentrarsi sulla popolazione sintomatica, che ritarda il miglioramento di qualsiasi proiezione a causa del periodo di incubazione immediato al contagio e, quindi, confonde la reale percezione del quadro epidemiologico - si ritiene probabile che il passaggio dallo Scenario $D$ allo Scenario $C$ sia possibile. Se questa transizione sarà confermata, le prospettive di cartolarizzazione della città attraverso la suddivisione in zone del traffico di persone e merci saranno inevitabili (modello di confinamento di Wuhan rafforzato dalla restrizione al traffico intrazonale) per raggiungere l'auspicabile restrizione del $60 \%-75 \%$, notando che la mappatura dei casi da la regione sarebbe determinante positivamente nell'aiutare a contenere la trasmissione della comunità in modo più accurato e osservare sia le persone contaminate che quelle anziane. Decisivi in tal senso sono l'avanzamento e la massificazione dei test, la distanza sociale, la prioritizzazione della localizzazione dei pazienti anziani e comorbosi, nonché l'isolamento di quelli diagnosticati (ZORZETTO, 2020).

RC: 80639

Disponibile in: https://www.nucleodoconhecimento.com.br/salute/previsione-dellapropagazione 


\section{MODELLAZIONE VSIR PER LA VALUTAZIONE DELLA CURVA DEI CASI ACCUMULATI DI COVID-19 IN AMAPÁ}

La serie storica con casi confermati di covid-19 per lo Stato di Amapá appartiene alla sezione dal 25 marzo al 12 maggio. In questo periodo, lo Stato di riferimento ha presentato la seguente evoluzione di individui risultati positivi, mortali e guariti:

Figura 2 Casi confermati di covid-19 (A). Evoluzione della mortalità (B). Casi recuperati $(\mathrm{C})$.

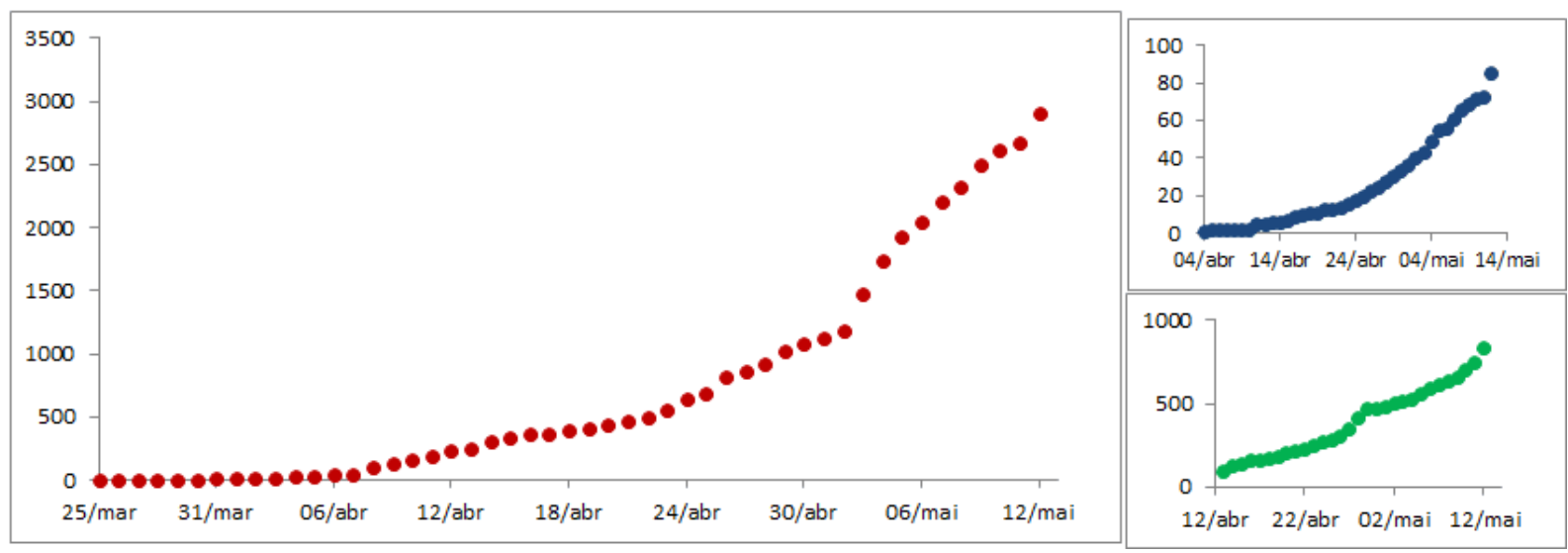

Come mostrato nella figura 2, in (A), Amapá ha impiegato 50 giorni per lasciare 1 caso per 2910 (dal 25 marzo al 12 Maggio Alla data di chiusura dello studio, lo stato aveva uno dei più alti tassi relativi di casi con circa 355,3 per 100.000 abitanti, attirando l'attenzione dell'intero paese perché è uno stato con meno di 1 milione di abitanti.

Sulla base di questo scenario, all'inizio dell'evoluzione temporale epidemica per Amapá, possiamo assumere $S \cong N$. Quindi, l'equazione per $d l / d t$ nel sistema (1) diventa:

RC: 80639

Disponibile in: https://www.nucleodoconhecimento.com.br/salute/previsione-dellapropagazione 


$$
\frac{d I}{d t} \cong(\beta-\gamma) I=\gamma\left(R_{0}-1\right) I
$$

Dove $R_{0}=\beta / Y$.. Dopo un'integrazione diretta otteniamo

$$
I(t) \cong I_{0} e^{\left(R_{0}-1\right) \gamma t}
$$

Per ottenere una prima stima di $R_{0}$ applichiamo una linearizzazione dell'equazione precedente. In questo modo otteniamo $\log (I(t))=m t+\log \left(I_{0}\right)$, dove abbiamo la pendenza $m=\gamma\left(R_{0}-1\right)$. L'adattamento con gli strumenti statistici del linguaggio di programmazione $R$ fornisce $m=0.21547 \pm 0.01163$. Sulla base della letteratura (TODA, 2020; LI, 2020; READ, 2020) assumiamo y nell'intervallo [0.1,0.6]. Con questo abbiamo una stima preliminare di $R_{0}$ tra [1.340, 3.271] che è un intervallo confrontabile con i valori riportati nelle tabelle riassuntive degli articoli di revisione su $R_{0}$ (LIU, 2020; ALIMOHAMADI, 2020).

Per quanto riguarda la fase di crescita esponenziale dei casi covid-19, nella Figura 3 c'è un soddisfacente accordo tra i dati osservati e la previsione teorica (curva rossa) fino al 14 aprile. Tuttavia, subito dopo c'è una discrepanza iniziale del fit preliminare con i dati, il che solleva interrogativi sul motivo di tale comportamento. Una possibile giustificazione di questo cambiamento di tendenza deriva dalla percezione che il periodo coincida con l'inizio delle politiche pubbliche per evitare il contagio. Tra questi c'è il Decreto n. 1497 del 3 aprile 2020 del Governo dello Stato di Amapá, che determina immediatamente l'isolamento sociale e la sospensione dei servizi considerati agglomerati fisici, con conseguente nuovo orario di apertura e mantenimento solo degli stabilimenti di estrema necessità aperto. Pertanto, si osserva che le misure di isolamento hanno un impatto sul numero di casi, dopo il ritardo di sedici giorni relativo al periodo di incubazione.

RC: 80639

Disponibile in: https://www.nucleodoconhecimento.com.br/salute/previsione-dellapropagazione 
Figura 3. Crescita esponenziale iniziale.

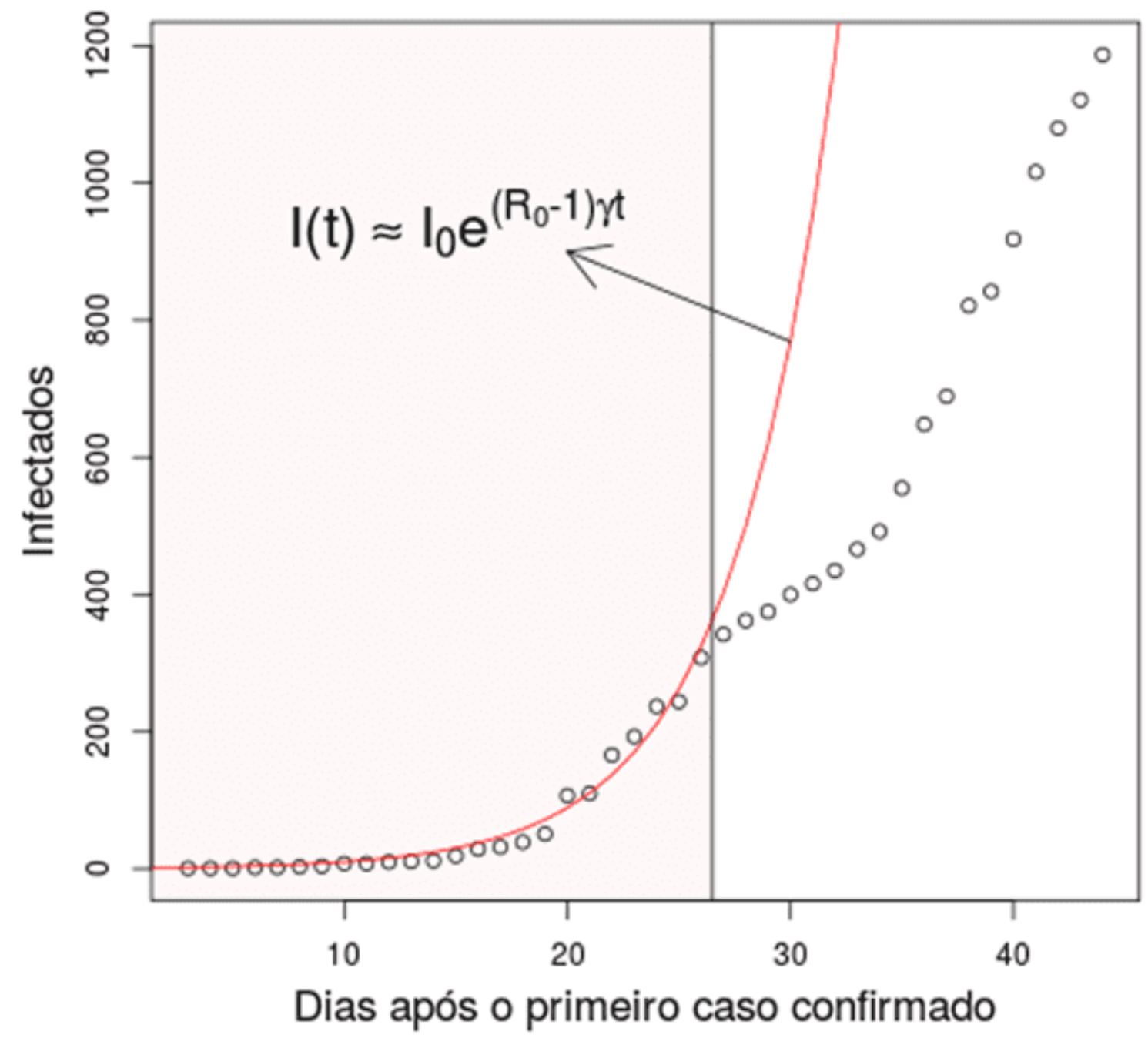

Mentre la figura 3 presenta un'analisi a breve termine con aggiustamento esponenziale (da 25 marzo a 14 aprile), nelle analisi successive i dati confermati dell'epidemia vengono confrontati con il modello matematico SIR standard e la variante impiegata da Batista (2020) (che chiamiamo vSIR, per brevità).

RC: 80639

Disponibile in: https://www.nucleodoconhecimento.com.br/salute/previsione-dellapropagazione 
Figura 4. Simulazione VSIR e intervallo di confidenza.

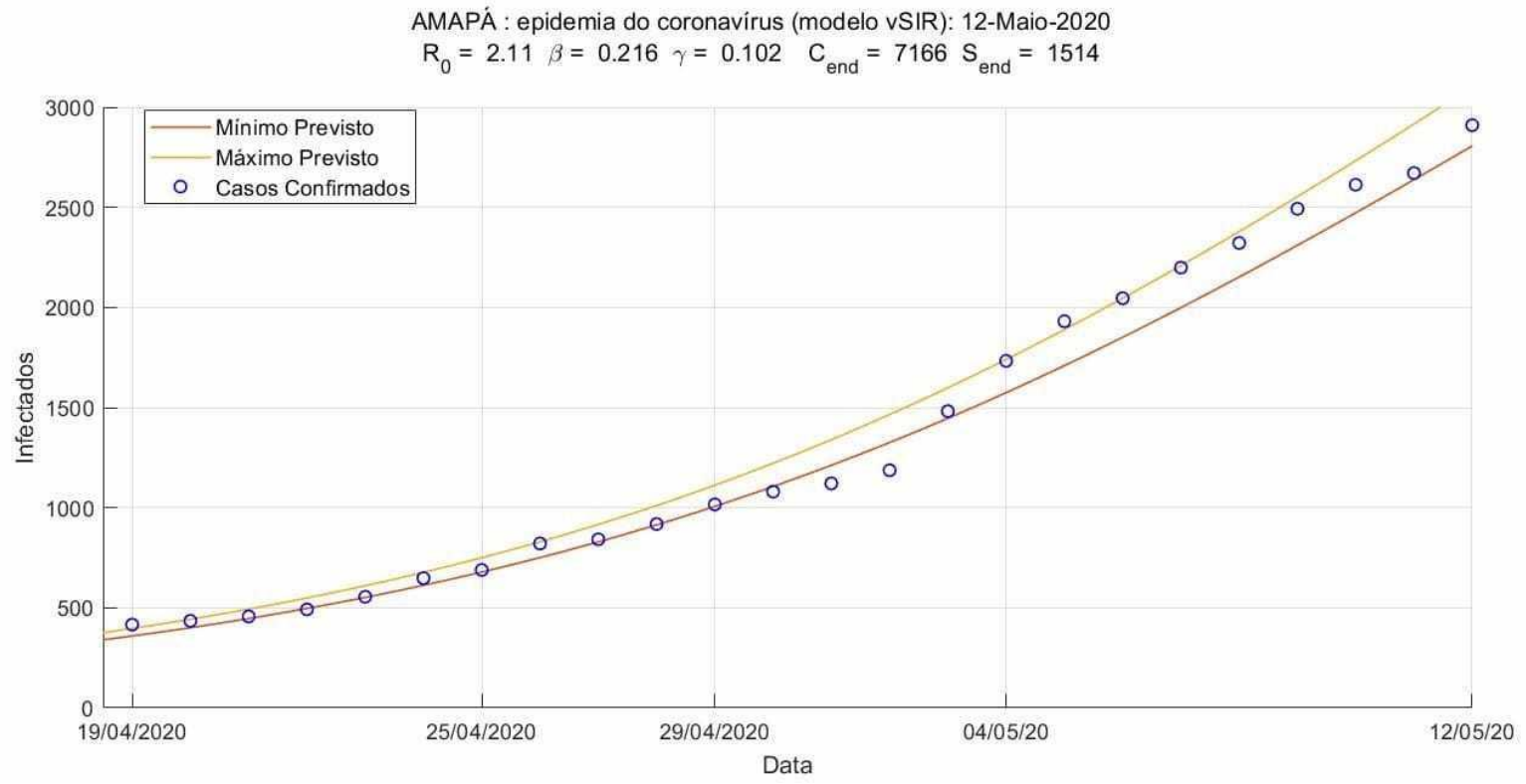

La simulazione del modello vSIR dal 25.03 al 12.05 .2020 mostrata in Figura 03 mostra l'intervallo di confidenza (IC) che definisce i limiti inferiore e superiore dei valori simulati al fine di dare una probabilità del $95 \%$ di includere il valore reale di casi predittivi. Ci sono due punti anomali che si verificano rispettivamente il 1 maggio 2020 e il 2 maggio 2020, venerdì e sabato. Queste date che riguardano la festa nazionale della festa dei lavoratori e il cui numero di contagiati non corrisponde alle aspettative sollevano interrogativi che coinvolgono il metodo procedurale di raccolta delle informazioni che verranno approfondite successivamente da un'analisi dei dati in loco. È anche interessante notare che il modello è stato calibrato sulla base dei dati effettivi delle persone infette dall'inizio della pandemia nello stato.

RC: 80639

Disponibile in: https://www.nucleodoconhecimento.com.br/salute/previsione-dellapropagazione 
Figura 5. Curva di previsione dei casi fino al 31.05.2020

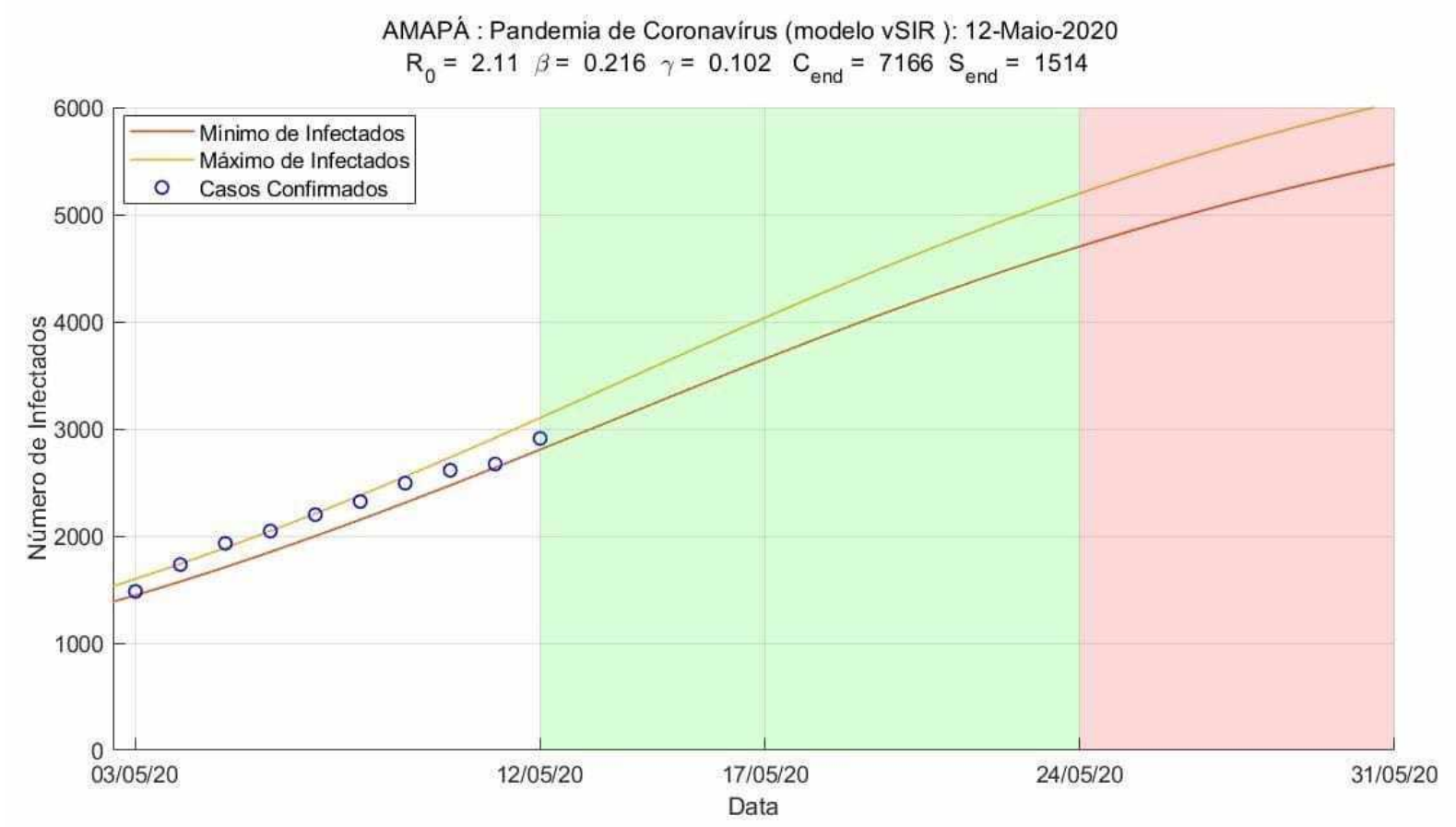

È possibile osservare in figura 5 la curva di previsione dei casi Covid-19 nello stato fino al giorno 31.05. Sembra che, fino al giorno 17.05 (domenica), il modello stima che lo stato avrà il numero di casi infetti vicino a 4mila (3651-4036), nella settimana successiva il giorno 24,05 questo numero sarà vicino al 5.000 casi (4700-5196), e infine fino alla fine di maggio, il 31 maggio, questi valori ruotano intorno a 5.500 casi (5469-6044). che è quasi il doppio di quanto registrato il 12.05. L'area verde da 12.05 a 24.05 rappresenta le date in cui il modello è più efficace nella previsione (date di chiusura), mentre l'area rossa rappresenta le date in cui il modello può fallire nella previsione (BATISTA, 2020).

I parametri stimati per i dati Amapá, utilizzando la programmazione fminsearch di MATLAB, sviluppata da Batista (2020), sono stati i seguenti:

RC: 80639

Disponibile in: https://www.nucleodoconhecimento.com.br/salute/previsione-dellapropagazione 
Tabella 6. Parametri stimati nel modello Vsir.

\begin{tabular}{ccc}
\hline$R_{0}$ & $\beta$ & $\gamma$ \\
2.11 & 0.216 & 0.102 \\
\hline
\end{tabular}

II valore stimato del tasso di riproduzione di base $R_{0}$ è in accordo con i valori riportati dalla letteratura specializzata nella revisione sistematica e nei metadati come in Liu (2020) e AliMohamadi (2020). Nel grafico di figura 04 si osserva anche un valore $=$ 7166, che è una proiezione del numero di casi per i giorni successivi ai dati disponibili. Nonostante il nome, non si riferisce al numero finale atteso se i dati disponibili sono ancora nelle fasi iniziali e intermedie dell'evoluzione epidemica, così come la situazione attuale dei dati nell'AP. Come vedremo di seguito, il modello vSIR fornisce previsioni a 5-10 giorni in linea con i dati osservati.

Nella tabella 7 è possibile osservare i valori previsti dei casi, con un IC del 95\%. Vale la pena ricordare che questi valori sono validi se non vi è alcun cambiamento nelle politiche pubbliche, o negli atteggiamenti individuali significativi, verso misure più severe in relazione allisolamento.

RC: 80639

Disponibile in: https://www.nucleodoconhecimento.com.br/salute/previsione-dellapropagazione 
Tabella 7. Previsione del numero di infetti al giorno.

\begin{tabular}{ccc}
\hline Data & $\begin{array}{c}\text { Intervalo de casos previstos } \\
\text { Mínimo }\end{array}$ & Máximo \\
\hline $\mathbf{1 3 . 0 5 . 2 0 2 0}$ & 2975 & 3288 \\
$\mathbf{1 4 . 0 5 . 2 0 2 0}$ & 3146 & 3477 \\
$\mathbf{1 5 . 0 5 . 2 0 2 0}$ & 3316 & 3665 \\
$\mathbf{1 6 . 0 5 . 2 0 2 0}$ & 3485 & 3852 \\
$\mathbf{1 7 . 0 5 . 2 0 2 0}$ & 3651 & 4036 \\
$\mathbf{1 8 . 0 5 . 2 0 2 0}$ & 3815 & 4216 \\
$\mathbf{1 9 . 0 5 . 2 0 2 0}$ & 3975 & 4394 \\
$\mathbf{2 0 . 0 5 . 2 0 2 0}$ & 4130 & 4565 \\
$\mathbf{2 1 . 0 5 . 2 0 2 0}$ & 4281 & 4732 \\
$\mathbf{2 2 . 0 5 . 2 0 2 0}$ & 4427 & 4893 \\
$\mathbf{2 3 . 0 5 . 2 0 2 0}$ & 4567 & 5048 \\
$\mathbf{2 4 . 0 5 . 2 0 2 0}$ & 4700 & 5196 \\
$\mathbf{2 5 . 0 5 . 2 0 2 0}$ & 4829 & 5338 \\
$\mathbf{2 6 . 0 5 . 2 0 2 0}$ & 4951 & 5472 \\
$\mathbf{2 7 . 0 5 . 2 0 2 0}$ & 5055 & 5600 \\
$\mathbf{2 8 . 0 5 . 2 0 2 0}$ & 5176 & 5721 \\
$\mathbf{2 9 . 0 5 . 2 0 2 0}$ & 5280 & 5835 \\
$\mathbf{3 0 . 0 5 . 2 0 2 0}$ & 5377 & 5944 \\
$\mathbf{3 1 . 0 5 . 2 0 2 0}$ & 5469 & 6044 \\
\hline
\end{tabular}

Fonte: prodotto dagli autori con dati di ricerca.

Considerando la tabella di previsione dei casi accumulati di infetti da covid-19 ad Amapá, il 31 maggio 2020, il modello prevede che lo Stato possa avere tra 5469 e

RC: 80639

Disponibile in: https://www.nucleodoconhecimento.com.br/salute/previsione-dellapropagazione 
6044 casi accumulati. Tenendo conto dell'attuale $p$-proporzione di letalità calcolata per questa unità federativa che il $12^{\circ}$ (ultimo giorno di dati effettivi raccolti) il suo valore era $p=2,95 \%$. Mantenendo questo livello, il 31 maggio potremmo avere tra 161 e 178 decessi a causa della malattia.

Per quanto riguarda il numero base di riproduzione $R_{0}$ il modello vSIR ha anche generato una serie temporale per il monitoraggio giornaliero dell'evoluzione del monitoraggio giornaliero $R_{0}$ finalizzato a conoscere l'entità dettagliata dell'evoluzione della malattia, oltre ad essere un indicatore del pubblico non farmaceutico politiche per la mitigazione della malattia COVID-19.

Figura 6 Evoluzione giornaliera di $R_{0}$.

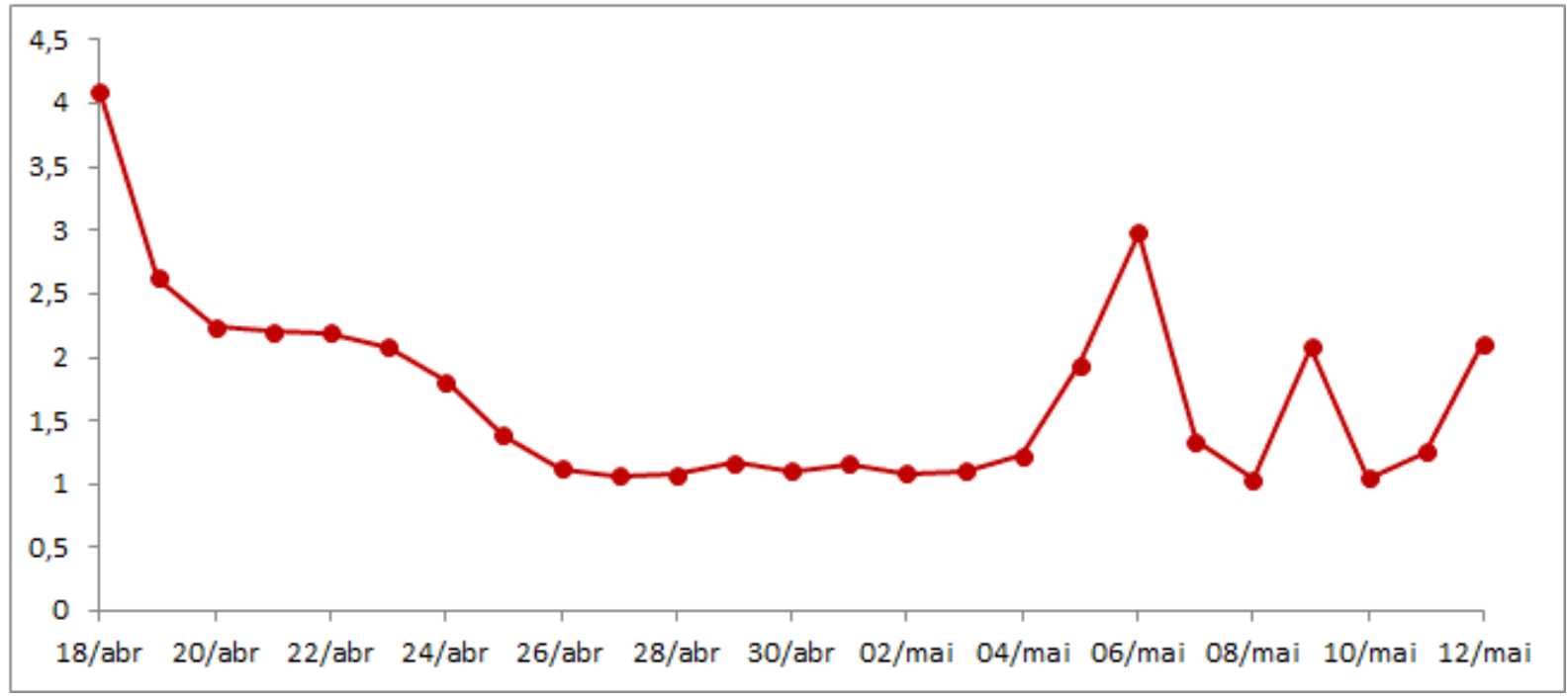

L'evoluzione giornaliera di $R_{0}$ mostra che i valori più bassi si sono verificati tra il 26 aprile $\left(R_{026 / 04}=1.12\right)$ al 3 maggio $\left(R_{003 / 05}=1.1\right)$ questo periodo di basso $R_{0}$ potrebbe indicare che il decreto governativo del 03 aprile ha contribuito a ridurre il tasso di riproduzione di base, ma non abbastanza da portarlo a meno di 1, che è l'ideale per controllare l'epidemia. Dal 3 maggio $R_{0}$ è nuovamente salito, essendo sul 12/5 al livello di 2.11, questo aumento può indicare un allentamento della quarantena da parte della popolazione, il cui rapporto con $R_{0}$ è inverso, riducendo la quarantena,

RC: 80639

Disponibile in: https://www.nucleodoconhecimento.com.br/salute/previsione-dellapropagazione 
aumentando $R_{0}$ e viceversa. versa, come riportato in Dandekar e Barbastathis (2010).

\section{LIMITAZIONI DELLO STUDIO}

Mentre nel modello SIR ci sono solo due parametri da stimare (ovvero la velocità di trasmissione $\beta$ e il recupero $\gamma$ ), nel modello vSIR abbiamo la possibilità di stimare un ulteriore parametro che è la dimensione effettiva della popolazione $N$. Con questi tre parametri $\{\beta, \gamma, N\}$ siamo stati in grado di descrivere in modo soddisfacente le serie temporali per il numero di casi confermati nello stato di Amapá.

La nostra modellazione può anche essere migliorata in lavori futuri considerando le seguenti limitazioni:

- Esiste una limitazione dell'applicazione lineare e generale del Modello ICL, da un lato, e dall'altro, eventuali inesattezze devono essere osservate a causa dell'interdipendenza e complessità delle condizioni specifiche dello sviluppo umano in Brasile - già menzionate, tali come concentrazione demografica accentuata in dozzine di città, condizioni precarie di servizi igienico-sanitari di base nelle comunità svantaggiate, abitazioni con residenti per stanza al di sopra di quanto raccomandato dall'Organizzazione Mondiale della Sanità (OMS), assenza storica di altre esperienze di emergenza sanitaria con misure di queste dimensioni e standard. In altre parole, il Brasile ha una proporzione specifica e condizioni demografiche, che aumentano l'imprecisione dei modelli generali.

- La sottostima dei casi di covid-19 è un fattore che è stato segnalato più volte in tutto il mondo, che nel caso brasiliano, a causa della ridotta quantità di test, rende critica l'incidenza di questo fattore. Ad esempio, recentemente circa $86 \%$ (intervallo di confidenza al 95\%: 82-90\%) della sottostima è stato stimato nelle prime fasi dell'evoluzione epidemica quando le restrizioni di viaggio non erano ancora state imposte (LI, 2020). Per Amapá, ci sono stime

$\mathrm{RC}: 80639$

Disponibile in: https://www.nucleodoconhecimento.com.br/salute/previsione-dellapropagazione 
che proiettano un tasso di sottostima del 11,2\%, secondo una nota tecnica pubblicata l'11 aprile (BATISTA et al, 2020b)

- Il modello vSIR non tiene conto del numero di casi asintomatici. Ad esempio, nell'articolo di revisione di (BUITRAGO-GARCIA, 2020) è stato stimato un limite superiore per la percentuale di infezioni asintomatiche SARS-CoV-2 di 29\% (intervallo di confidenza al 95\%: 23-37\%). Una strategia per ottenere una stima della frazione di casi asintomatici in Amapá consiste nell'investire nel testare una quota massiccia della popolazione.

\section{CONCLUSIONI}

Nel primo approccio, è stata sviluppata una proiezione comparativa lineare del modello ICL per COVID-19 applicato al Brasile per Amapá, le cui conclusioni indicano l'urgenza delle prime misure possibili di isolamento sociale, considerando la necessità di evitare un contagio massiccio e accelerato in la località. Abbiamo anche cercato di osservare le condizioni generali del contesto pandemico. Esiste un'imprecisione per quanto riguarda la velocità effettiva di trasmissione e infezione, sebbene circondata da un certo grado di misurazione, mesi dopo l'inizio di una pandemia dichiarata, implica direttamente l'osservazione dei rispettivi standard locali.

Il secondo approccio è stato mediato dalla soluzione a breve termine delle equazioni del modello SIR. Questa soluzione ha fornito la tipica crescita esponenziale che si è dimostrata in accordo con i dati osservati fino al 14 aprile, consentendo di ottenere una stima preliminare di $R_{0}$ compresa tra [1.340, 3.271]. Nei giorni successivi si riscontra una brusca e precoce discrepanza dell'esponenziale fit dovuta alle misure di isolamento adottate.

Nel terzo approccio, il modello vSIR è stato applicato ai dati infetti effettivi. È stato verificato che il tasso di riproduzione di base $R_{0}$ per Amapá è 2,11, a dimostrazione che l'epidemia in questo stato è in piena espansione, questo fatto è ancora più aggravato dall'informazione dell'isolamento sociale che mostra che lo Stato, alla luce

$\mathrm{RC}: 80639$

Disponibile in: https://www.nucleodoconhecimento.com.br/salute/previsione-dellapropagazione 
del primo II decreto di quarantena ha raggiunto il $56 \%$ dei suoi cittadini isolati il 5 aprile, tuttavia, quel numero è sceso al $43 \%$ il 12 aprile.

Affinché ci sia una riduzione del livello di controllo dell'epidemia $\left(R_{0}<1\right)$, è necessario che la velocità di trasmissione $\beta$, che ora è a $\sim 0,216$, subisca una riduzione di oltre il $53 \%$ per avere $R_{0}$ sotto controllo, che è possibile solo con azioni rigorose per controllare la dispersione della popolazione per ridurre i contatti tra gli individui, rallentando così l'interazione tra individui suscettibili e infettivi.

\section{GRAZIE}

Gli autori sono particolarmente grati a Milan Batista da University of Ljubljana per i suoi utili contributi nell'uso del codice open source per Matlab e per il supporto di CAPES e CNPQ nello sviluppo di questo lavoro, attraverso la concessione di una borsa di studio di dottorato e sostegno alla ricerca.

\section{RIFERIMENTI}

ALIMOHAMADI, Yousef; TAGHDIR, Maryam; SEPANDI, Mojtaba. The estimate of the basic reproduction number for novel coronavirus disease (covid-19): A systematic review and meta-analysis. Journal of Preventive Medicine and Public Health, 2020. Disponível em: <https://www.jpmph.org/upload/pdf/jpmph-20-076.pdf> Acesso em: 01/05/2020

AMAPÁ. Painel Coronavírus. [internet].2020a. Disponível em: <http://painel.corona.ap.gov.br/ > Acesso em: 10/05/2020

AMAPÁ. Superintendência de Vigilância em Saúde - SVC. 2020b. Disponível em: $<$ https://svs.portal.ap.gov.br/coronavirus> Acesso em: 14/04/2020.

RC: 80639

Disponibile in: https://www.nucleodoconhecimento.com.br/salute/previsione-dellapropagazione 
BATISTA, Milan. fitVirusCOVID19. MATLAB Central File Exchange. [Internet]. 2020. Disponível em: <https://www.mathworks.com/matlabcentral/fileexchange /74658fitviruscovid19> Acesso em 15/05/2020.

BROOKS, Samantha K.; WEBSTER, Rebecca K.; SMITH, Louise E.; WOODLAND, Lisa.; WESSELY, Simon; GREENBERG, Neil. RUBIN, Gideon James. The psychological impact of quarantine and how to reduce it: rapid review of the evidence. The Lancet. London, Uk, v. 395, p.912-920, mar. 2020. DOI:https://doi.org/10.1016/S0140-6736(20)30460-8

BUITRAGO-GARCIA, Diana C.; EGLI-GANY, Dianne; COUNOTTE , Michel J.; HOSSMANN, Stefanie; IMERI, Hira; SALANTI, Georgia. LOW, Nicola. The role of asymptomatic SARS-CoV-2 infections: rapid living systematic review and metaanalysis. medRxiv, 2020. Disponível em: $<$ https://www.medrxiv.org/content/10.1101/2020.04.25.20079103v1> Acesso em: $10 / 05 / 2020$

DANDEKAR, R.; BARBASTATHIS, G. Neural Network aided quarantine control model estimation of COVID spread in Wuhan, China. 2020. Disponível em: <https://arxiv.org/abs/2003.09403> Acesso em: 10/05/2020.

DELAMATER, Paul L.; STREet, Erica J.; LESLIE, Timothy F., YANG, Y. Tony; JACOBSEN, Kathryn H. Complexity of the Basic Reproduction Number (R0). Emerg Infect Dis 2019;25:1-4 PMC6302597

FERGUSON, Neil M.; GHANI, Azra C.; WALKER, Patrick G. T.; WHITTAKER, Charles; WATSON, Oliver; BAGUELIN, Marc; AINSLIE, Kylie E. C. et al.. The Global Impact of COVID-19 and Strategies for Mitigation and Suppression. 26 mar 2020. Imperial College London COVID-19 Response Team. Disponível em: $<$ https://www.imperial.ac.uk/media/imperial-college/medicine/mrc-gida/2020-03-26COVID19-Report-12.pdf> Acesso em: 14/05/2020.

RC: 80639

Disponibile in: https://www.nucleodoconhecimento.com.br/salute/previsione-dellapropagazione 
HARKO, Tiberiu; LOBO, Francisco S. N.; MAK, M. K. Exact analytical solutions of the Susceptible-Infected-Recovered (SIR) epidemic model and of the SIR model with equal death and birth rates. Applied Mathematics and Computation. 236: 184194, 2014. Disponível em: <https://arxiv_org/abs/1403.2160> Acesso em: 10/05/2020

HETHCOTE, Herbert W. The mathematics of infectious diseases. SIAM Review, v. 42, n. 4, p. 599-653, 2000. Disponível em: <https://epubs.siam.org/doi/abs/10.1137/s0036144500371907> Acesso em: $10 / 05 / 2020$

IBGE. Cidades e Estados: Amapá. 2020. [internet]. Disponível em: <https://www.ibge.gov.br/cidades-e-estados/ap.html> Acesso em: 20.04.20

IN LOCO. Mapa de Dados. 2020. [internet]. Disponível <https://mapabrasileirodacovid.inloco.com.br> Acesso em 03/05/2020.

MRAD, Assaad; BONETTI, Sara; MANOLI, Gabriele; PAROLARI, Anthony J. Global convergence of COVID-19 basic reproduction number and estimation from early-time SIR dynamics. 2020.2 DedRxiv. Disponível <https://www.medrxiv.org/content/10.1101/2020.04.10.20060954v2> Acesso em: $01 / 05 / 2020$

LI, Ruiyun; PEI, Sen; CHEN, Bin; YIMENG, Canção; ZHANG, Tao; YANG, Wan; SHAMAN, Jeffrey. Substantial undocumented infection facilitates the rapid dissemination of novel coronavirus (SARS-CoV-2). Science, v. 368, n. 6490, p. 489493, 2020. Disponível em: <https://science.sciencemag.org/content/368/6490/489> Acesso em: 10/05/2020

LIU, Ying; GAYLE, ALBERT A.; WILDER-SMITH, Annelies; ROCKLÖV Joacim. The reproductive number of COVID-19 is higher compared to SARS coronavirus. Journal of travel medicine, 2020. Disponível em:

RC: 80639

Disponibile in: https://www.nucleodoconhecimento.com.br/salute/previsione-dellapropagazione 
<https://academic.oup.com/jtm/article/27/2/taaa021/5735319>

Acesso

em: $10 / 05 / 2020$

MINISTÉRIO DA SAÚDE. Sobre a Doença Covid-19. Brasília. 2020. [internet]. Disponível em <https://coronavirus.saude.gov.br/sobre-a-doenca> Acesso em: $14 / 05 / 2020$

MINISTÉRIO DA SAÚDE. Síntese de casos, óbitos, incidência e mortalidade. Brasília. 2020a. [internet]. Disponível em: <https://covid.saude.gov.br/> Acesso em $15 / 05 / 2020$.

ORGANIZAÇÃO MUNDIAL DA SAÚDE. Coronavirus disease 2019 (COVID-19) Situation Report - 46. Disponível em <https://www.who.int/docs/defaultsource/coronaviruse/situation-reports/20200306-sitrep-46-covid19.pdf?sfvrsn=96b04adf_4\#: :text=For\%20COVID\%2D19\%2C,infections\%2C\%20re quiring\%20ventilation.> Acesso em 16/05/2020

PIRES, L.N.; CARVALHO, L.; XAVIER, L.L. COVID-19 e Desigualdade no Brasil. Centro Brasileiro de Estudos da Saúde. Disponível em < http://cebes.org.br/2020/04/covid-19-e-desigualdade-no-brasil/>. Acesso em 16/05/2020.

PRADO, Marcelo; BASTOS, Leonardo; BATISTA, Amanda; ANTUNES, Bianca; BAIÃO, Fernanda, MAÇAIRA, Paula; HAMACHER, Silvio; BOZZA, Fernando. Nota Técnica 7 NOIS/Puc-Rio: Análise de subnotificação do número de casos $\begin{array}{lllll}\text { confirmados } & \text { da } & \text { COVID-19 } & \text { no }\end{array}$ <http://www.supersuporte.com/myRpubs/NT7_Subnotificacao_notaDia11-abr2020.pdf>, Acessado em: 14.05.2020.

PRADO, Marcelo; BASTOS, Leonardo; BATISTA, Amanda; ANTUNES, Bianca; BAIÃO, Fernanda, MAÇAIRA, Paula; HAMACHER, Silvio; BOZZA, Fernando. Nota Técnica 7 NOIS/Puc-Rio: Evolução da epidemia de COVID-19 no Brasil até 04 de

$\mathrm{RC}: 80639$

Disponibile in: https://www.nucleodoconhecimento.com.br/salute/previsione-dellapropagazione 
maio

de

2020.

<https://drive.google.com/file/d/17a9F6WEmCoihJmXF3KMHEl1jWo51xarz/view>, Acesso em: 14/05/2020

READ, Jonathan M.; BRIDGEN, Jessica R. E.; CUMMINGS, Derek A. T. HO, Antonia; JEWELL, Chris P. Novel coronavirus 2019-nCoV: early estimation of epidemiological parameters and epidemic predictions. MedRxiv, 2020. Disponível em: <https://www.medrxiv.org/CONTENT/10.1101/2020.01.23.20018549V2> Acesso em $10 / 05 / 2020$

TODA, Alexis Akira. Susceptible-infected-recovered (sir) dynamics of covid-19 and economic impact. ArXiv:2003.11221, 2020. Disponível em: <https://arxiv.org/abs/2003.11221> Acesso em: 09/05/2020

ZHANG, Yuan; YOU, Chong; CAI, Zhenghao; SUN, Jiarui; HU, Wenjie; ZHOU, XiaoHua. Prediction of the COVID-19 outbreak based on a realistic stochastic model. medRxiv, $2020 . \quad$ Disponível em: <https://www.medrxiv.org/content/10.1101/2020.03.10.20033803v1> Acesso em: $09 / 05 / 2020$

ZHONG, Linhao; MU, Lin;LI, Jing; WANG, Jiaying; YIN, ZHE; LIU, Darong. Early prediction of the 2019 novel coronavirus outbreak in the mainland china based on simple mathematical model. leee Access, v. 8, p. 51761-51769, 2020. Disponível em: <https://ieeexplore.ieee.org/document/9028194> Acesso em: 09/05/2020

ZORZETTO, R. A segunda onda do coronavírus. PESQUISA FAPESP, 2020. Disponível em <https://revistapesquisa.fapesp.br/2020/03/30/a-segunda-onda-docoronavirus/> Acesso em: 09/05/2020

\section{APPENDICE - RIFERIMENTO A PIEDI}

11. http://www2.unifap.br/matematicaplicada/pagina-exemplo/

$\mathrm{RC}: 80639$

Disponibile in: https://www.nucleodoconhecimento.com.br/salute/previsione-dellapropagazione 
Inserito: Maggio, 2020.

Approvato: Maggio, 2020.

RC: 80639

Disponibile in: https://www.nucleodoconhecimento.com.br/salute/previsione-dellapropagazione 